\title{
Montreal Computer Chess Workshop
}

Montreal, PQ, Canada

August 13-14, 1982

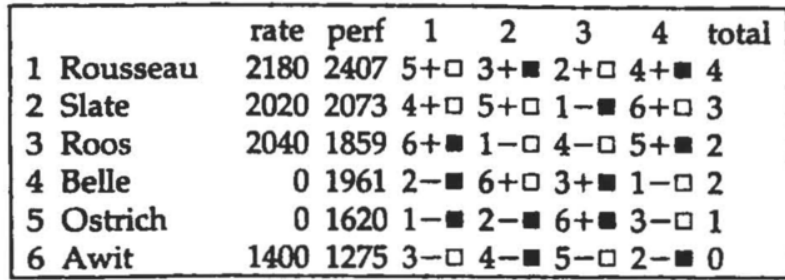

\section{Rousseau - Ostrich}

1 e4 d5 2 exd5 $₫$ f6 3 c4 c6 4 d4 cxd5 5 ¿c3 e6 6 \&g5 2 b4 7 बी 3 O-O 8 cxd5 exd5 9

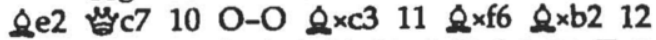

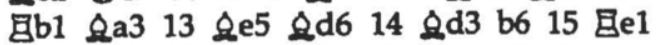

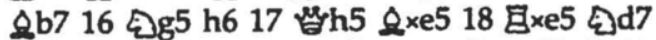

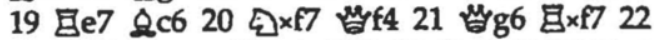

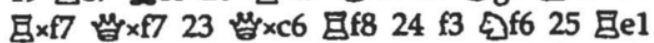
h5 26 h4 this 27 目e5 쌈g8 28 쌈7 目f7 29

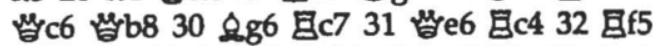
Exd4 33 IXf6 目d1+ 34 कीf2 目d2+ 35 te3

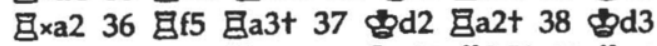

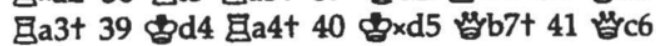

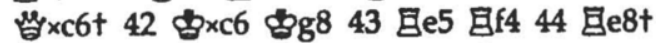

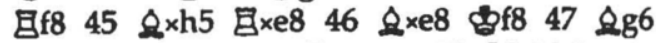
te7 48 6b7 b5 49 को $\times a 7$ b4 50 कb6 b3 1-0

$$
\text { Slate - Belle }
$$

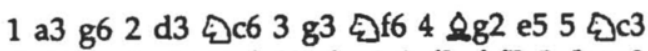

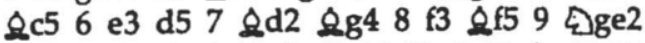
目f8 10 e4 2 e6 11 \&g5 d4 12 @d5 $2 \times d 513$

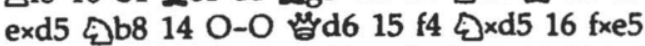

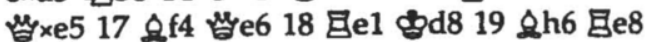

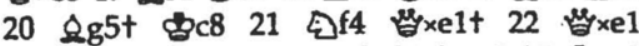

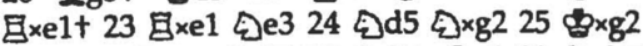

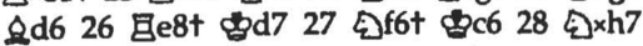

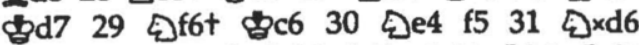

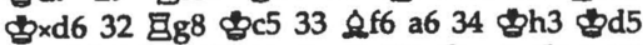
35 the b5 36 fg5 c5 37 किखg6 bc6 38 bxf5 bb7 39 \&e5 a5 40 h4 ¿c6 1-0

$$
\text { Awit - Roos }
$$

1 b3 $Q$ f6 2 Sb2 e6 3 e3 d5 4 \&f3 \&e7 5

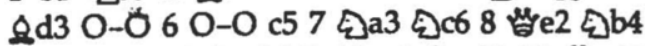

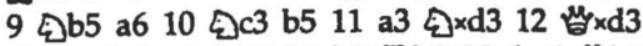

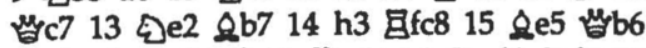

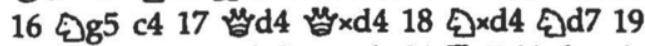

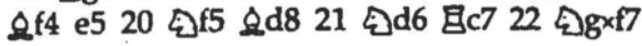

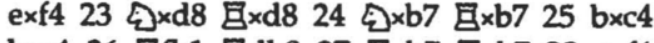
b×c4 26 目fb1 目db8 27 Exb7 若×b7 28 exf4

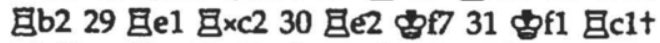

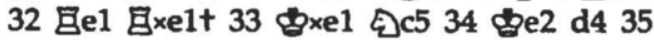

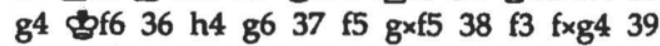
fxg4 te5 40 bf 3 h6 41 te2 toff 42 g5 h×g5 43 h5 छोe6 44 d3 c3 45 h6 ¿f8 46 a4 g4 47 a5 g3 48 gel c2 49 gd2 o-1

$$
\text { Roos - Rousseau }
$$

1 c4 Ðf6 2 Ðc3 c5 3 Ðf3 e6 4 d4 d5 5 cxd5

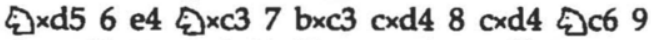

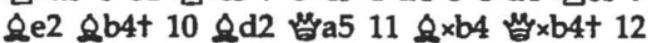

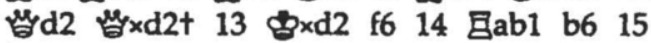
Ehc1 \& 8 d7 16 \&b5 Ic8 $17 \mathrm{~d} 5$ exd5 18 exd5

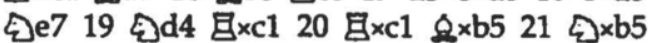

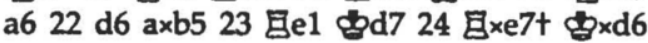

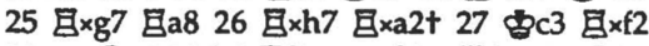

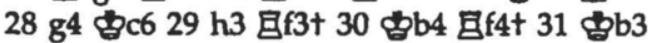

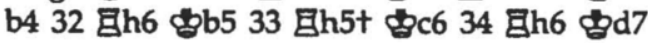
35 ba4 कोe7 36 gh5 tof7 37 gb5 gg6 38

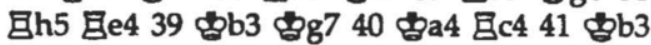
Ifc5 0-1

$$
\text { Ostrich - Slate }
$$

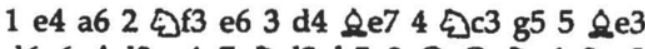
d6 6 \&d3 g4 7 छीd2 h5 8 O-O \&c6 9 a3 \&d7 $10 \mathrm{~h} 3$ e5 $11 \mathrm{dxe} 5$ छिxe5 $12 \mathrm{~h} \times \mathrm{g} 4 \mathrm{~h} \times \mathrm{g} 4$

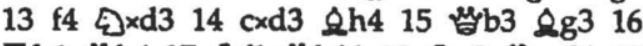

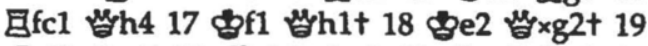

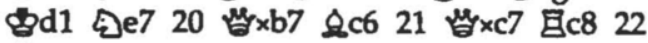

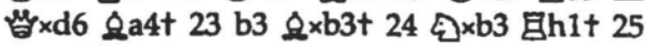
\&g1 目×g1\# 0-1

$$
\text { Belle - Awit }
$$

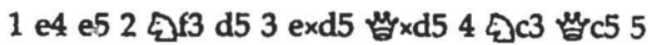

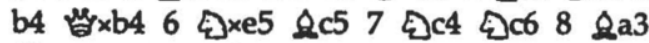

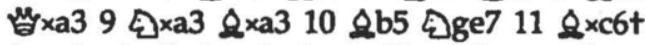

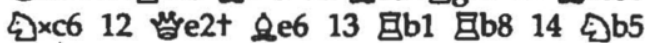




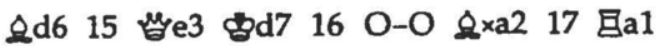

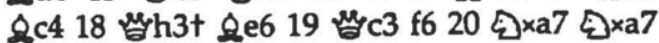

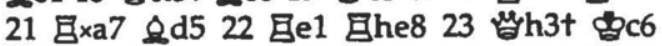

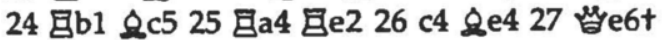

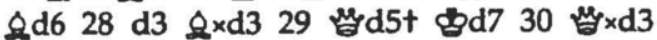

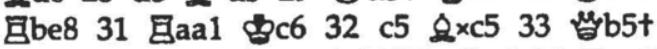
bd6 34 目d1十 the7 35 目d7t the6 36 目×97 \& $\times 2+37$ को 1 1-0

$$
\text { Rousseau - Slate }
$$

1 e4 c6 2 d4 d5 3 घd2 dxe4 4 ¿xe4 2 f6 5

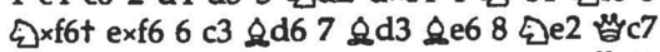
9 ¿g3 g6 10 ¿e4 \&e7 11 요e3 ఏd7 12 쌉d2 \&b6 13 \& 84 씸d7 $14 \mathrm{~h} 4$ \&d5 15 \&gg3 h6 16

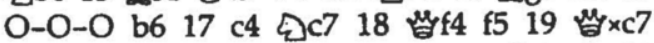

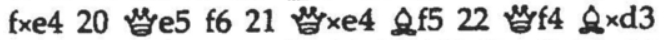
23 Bxd3 thf7 24 d5 f5 25 ghd1 c5 26 d6

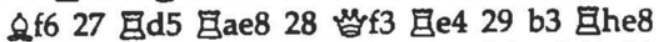
30 h5 @g5t 31 bb1 国2 32 \&f4 \&f6 33

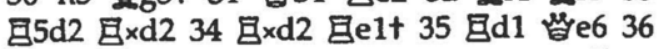
h×g6t dog6 37 doc2 a5 38 g3 a4 39 kd3 axb3t 40 axb3 kㅏㅁe8 $41 \mathrm{~g} 4$ 1-0

$$
\text { Roos - Belle }
$$

1 c4 e6 2 g3 @f6 3 \&g2 d5 4 b3 \&e7 5 صf3 d4 6 O-O O-O 7 Be1 ¿c6 8 e4 e5 9 d3

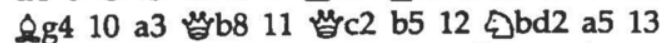

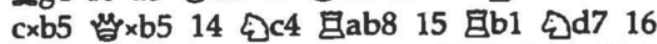
odd2 f5 17 ¿g5 f4 18 gxf4 exf4 19 \&f3 ․ㅏㅁh5

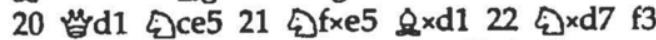

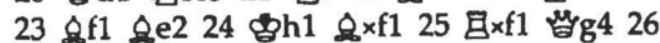

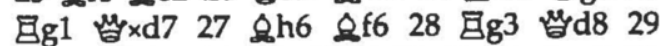

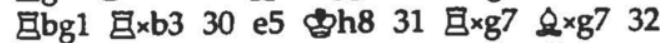

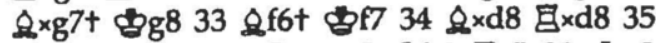

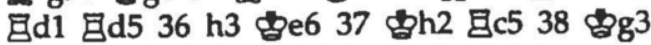

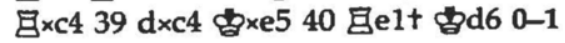

$$
\text { Awit - Ostrich }
$$

1 c4 巳f6 2 d4 d5 3 e3 \&g4 4 쌉b3 \& c8 5

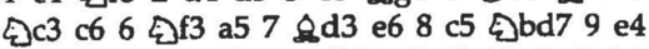
\&e7 10 exd5 cxd5 11 쌈b5 O-O 12 O-O h6

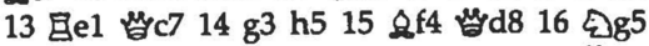

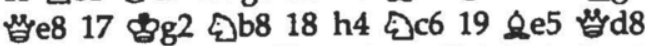

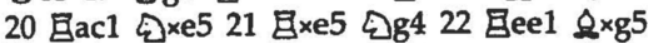

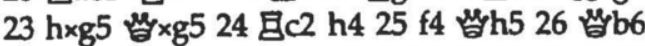
h3t 27 bh1 h2 28 gee2 k্vh3 29 Ig2 g6 30

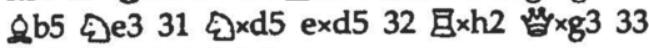
国1 애3 34 b3 0-1

$$
\text { 4h. Belle - Rousseau }
$$

1 e4 c5 2 c3 e6 3 d4 d5 4 exd5 exd5 5 ¿f3 \&c6 6 \&e3 c4 7 b3 cxb3 8 axb3 \&d6 9 冒4

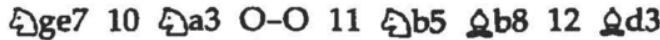

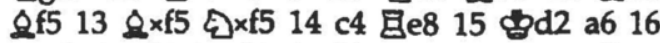

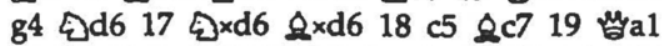

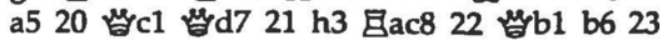

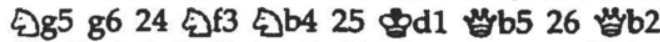
쌉d3t 27 घd2 bxc5 28 dxc5 d4 29 目 $\times b 4$ dxe3 30 目d4 빵b5 31 fxe3 요 32 쌈c2

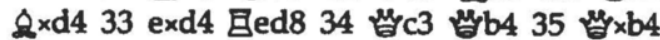
a×b4 36 e2 目×d4 37 目c1 目d5 38 巳e4 目e5 39 bd3 f5 40 gxf5 gxf5 41 国 1 t bf8 42 ¿g5 h6 43 bd4 目e7 44 目f1 h×g5 45 目×f5十

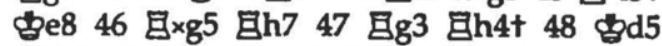
国h5t 49 bc4 国cxc5t 50 bxb4 目cf5 51 bc3 目4 52 bd2 0-1

$$
\text { Slate - Awit }
$$

1 h4 d6 2 a4 صc6 3 日a3 e5 4 目e3 \&e7 5 g3

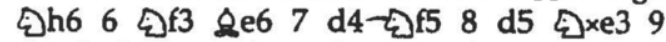

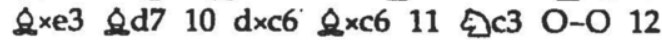
Qh3 国8 13 O-O \&f6 14 छd5 \&.a4 15

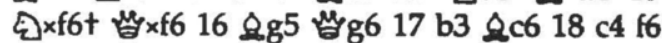
19 \&e3 a5 20 쌈d2 a4 21 b4 f5 22 乞g5 h6 23 b5 h×g5 24 bxc6 bxc6 25 ㅇg5 月eb8 26 国a1

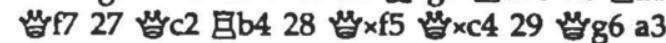

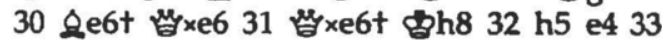

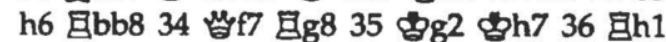
a2 $1-0$

$$
\text { Ostrich - Roos }
$$

1 e4 c6 2 d4 d5 3 e5 \&f5 4 ¿f3 e6 5 \&f4

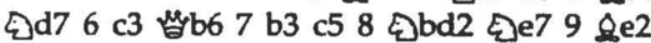
¿c6 $10 \mathrm{dxc5} \& \times c 511$ O-O O-O 12 b4 \&e7

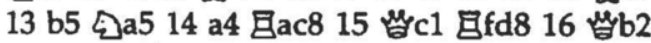

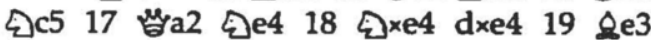

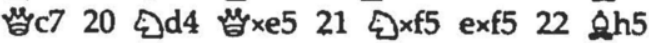

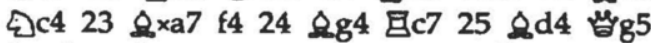

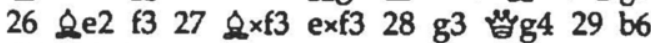
밥. 3 o-1 